\title{
Endovascular Instead of Open Surgical Repair of Axillosubclavian Artery Injuries: An Evolving Paradigm Shift
}

\author{
Shreya Jalali', Derek J Roberts², Megan L Brenner'3 , Joseph J DuBose4, \\ Laura J Moore ${ }^{5}$ and Adam H Power ${ }^{1}$ \\ 'Division of Vascular Surgery, Department of Surgery, Western University, London Health Sciences Centre, Victoria Hospital, \\ London, Ontario, Canada \\ 2Division of Vascular and Endovascular Surgery, Department of Surgery, University of Ottawa, The Ottawa Hospital, \\ Civic Campus, Ottawa, Ontario, Canada \\ ${ }^{3}$ Department of Surgery, University of California Riverside, Riverside, California, USA \\ ${ }^{4}$ R Adams Cowley Shock Trauma Center, University of Maryland School of Medicine, Maryland, USA \\ ${ }^{5}$ Department of Surgery, The University of Texas Health Science Center, Houston, Texas, USA
}

\begin{abstract}
Axillosubclavian injuries (ASI) comprise a small proportion of vascular injuries, yet their morbidity and mortality is high. This is often attributable to non-compressible bleeding in the apical thorax, hemodynamic instability, and the anatomically challenging location of these vessels making them difficult to access and control quickly. While the traditional management of ASI was with open surgical repair, recent years have seen an evolution towards less invasive endovascular repair (EVR). In patients with these injuries, EVR may be a safer alternative that achieves similar immediate results with significantly lower complication and mortality rates than the highly morbid open surgical option. In this article, we review and compare the two approaches, providing an overview of patient selection, anatomic considerations, techniques, postoperative management, and outcomes. With the advent of endovascular trauma management and more trauma team members capable of endovascular management of vascular trauma, a paradigm shift towards EVR for ASI is taking place.
\end{abstract}

Keywords: Axillosubclavian; Vascular Trauma; Endovascular; EVTM

Received: 5 October 2021; Accepted 7 October 2021

\section{INTRODUCTION}

Blunt or penetrating trauma to the axillosubclavian arterial segment comprises $5 \%$ of all vascular injuries $[1,2]$. These most often occur because of stab or gunshot wounds [3-5] and less commonly after motor vehicle collisions or falls from heights [6]. Concurrent injuries to the brachial plexus, pharynx or esophagus, and trachea, bronchi, and lung are common [1,7-10].

\section{Corresponding author:}

Adam H Power, Division of Vascular Surgery, Department of Surgery, Western University, London Health Sciences Centre, Victoria Hospital, London, Ontario, Canada. Room E2121. 800 Commissioners Rd East, London, Ontario, Canada, N6A 5W9.

Email: Adam.Power@lhsc.on.ca

(C) 2021 CC BY-NC 4.0 - in cooperation with Depts. of Cardiothoracic/Vascular Surgery, General Surgery and Anesthesia, Örebro University Hospital and Örebro University, Sweden
Blunt axillosubclavian injuries (ASI) are frequently associated with clavicle fractures, fractures of the first three ribs, and shoulder dislocations [6]. Patients can present with hemorrhage, diminished or absent upper limb pulses, arterial bruits, hematomas, or neurologic deficits [11].

Despite being relatively uncommon, the morbidity and mortality of ASI is high [6,11-13]. Mortality estimates in contemporary series remain as high as $20.5 \%$ [7] to $39 \%$ [4]. This is often secondary to noncompressible bleeding in the apical thorax, hemodynamic instability, and the frequently deep junctional location of these vessels, which makes them difficult to access and control quickly.

In this article, we review and compare endovascular and open surgical repair (OSR) of ASI. Specifically, we discuss patient selection, technical considerations, postoperative management, and outcomes of endovascular and open management of ASI. 


\section{Ethical Approval and Informed Consent}

Ethical approval was not required. Informed consent was not required.

\section{OPEN SURGICAL REPAIR}

The traditional approach to these injuries has been OSR. This often involved generous para-clavicular incisions $[14,15]$, sternotomy, or thoracotomy for exposure and proximal and distal control $[1,2,16]$. This is because of the anatomical constraints of obtaining surgical exposure in the apical thorax, a relatively small confined space densely packed with neurovascular structures such as the brachial plexus. If they have not already been injured, these structures are prone to inadvertent injury as the surgical dissection often occurs in a bleeding field with tissues that may have been distorted by hematoma.

In order to obtain control of a proximal left subclavian artery injury, a high anterolateral thoracotomy is often required. Proximal right subclavian or innominate artery injuries often require sternotomy for proximal control and exposure. These injuries can be primarily repaired or replaced with autogenous or prosthetic graft material depending on the extent of injury and contamination. Distal injuries are usually exposed more directly with control near the injury site and have been treated with direct repair, anatomic bypass, or extra-anatomic bypass, including axillary-brachial or carotid-brachial bypass [17].

Anatomical considerations that support OSR may include very long segment injuries, insufficient proximal or distal normal vessel fixation points for stents, and extensive total arterial transection [11]. In cases of severe uncontrolled bleeding, concurrent venous injury or transection, upper extremity compartment syndrome resulting in neurovascular compression, or concurrent injuries requiring OSR or debridement, OSR would usually be the first choice.

OSR of ASI is associated with a high risk of mortality ranging from $5 \%$ to $30 \%$ [18-20]. The mortality rates of OSR for penetrating injuries of the subclavian artery have been particularly high $[4,7,21,22]$. In one large retrospective study, the mortality of penetrating ASI was $34.2 \%$ overall and $14.8 \%$ for those that made it to the operating room [7]. It follows that this increased mortality could in large part be attributed to the hemodynamic instability that patients with penetrating ASI often present with.

OSR also has been reported to be associated with long operative durations, lengths of hospital stay, and a higher risk of postoperative complications [3,6,13]. A 10-year analysis of the National Trauma Databank identified 3,628 patients with ASI, of with only 9\% undergoing endovascular repair (EVR) versus OSR [3]. Complication rates were notably different when compared; for instance, surgical site infections occurred in $7 \%$ of OSR cases versus $4 \%$ of EVR, pneumonia in $8 \%$ of OSR versus $5 \%$ of EVR, and ICU admission in $31 \%$ of OSR versus $21 \%$ of EVR cases. Further, the overall mortality rate was significantly greater for OSR at $14.2 \%$ versus $8.8 \%$ for $\operatorname{EVR}(P=0.01)$. It should of course be noted that despite concluding that EVR was independently associated with lower odds of complications after controlling for confounding variables such as admission vitals and ISS, there is inextricable bias in that patients selected for EVR are generally already those likely to have more favorable outcomes.

\section{EVOLUTION OF EVR}

The inception of stents in the 1960s occurred when the first "endoluminal splint" was reported to be placed post-angioplasty to prevent recoil and dissection [23]. In 1991, Parodi et al. began to use the first stent-grafts covered with fabric for abdominal aortic aneurysm treatment [24]. Through the 1990s, stents for cardiovascular procedures gained significant traction with improvement of technologies and by the 2000s, EVRs had become mainstream.

Stent grafts have since been adopted in many trauma centers for use in arterial injuries as well, including of the brachiocephalic vessels, aorta, and lower extremity arteries $[25,26]$. They can be used as a first-line treatment for both blunt and penetrating injuries, and the remote approach avoids the morbid dissection described above while producing safe and effective immediate results thus far $[6,11,17,27]$. EVR is now increasingly being used as a viable management option even for critically ill, hemorrhaging patients with traumatic vascular injuries [28,29], consistent with the paradigm shift towards endovascular trauma management (EVTM) for hemodynamically unstable patients with vascular injuries [30]. It is important to note, however, that risk of perioperative rupture remains ever-present and during EVR one should always be prepared to convert to open surgical bypass or reconstruction if required. Branco and DuBose in 2016 found that, among 92 ASIs, 88 $(95.6 \%)$ had successful endovascular stent placement but $4(4.3 \%)$ required open conversion [31].

EVR of arterial injuries mitigates the significant risks associated with open repair, which is conceivably a major reason for its uptake in contemporary management of ASI. EVR of traumatic arterial injuries has been documented in aortic and iliac vessels as well as axillary and subclavian injuries. For ASI, the rates of EVR have been increasing significantly in recent years. In one review, from 2003 to 2013 the rate of EVR increased from $5.3 \%$ to $22.2 \%$ with the incidence of these injuries remaining unchanged [12]. EVR has been performed in penetrating, iatrogenic, and blunt injuries. Blunt injuries often present with multiorgan issues which require OSR to address, whereas EVR is best utilized for focal lesions that can be safely traversed with a guidewire [13]. 


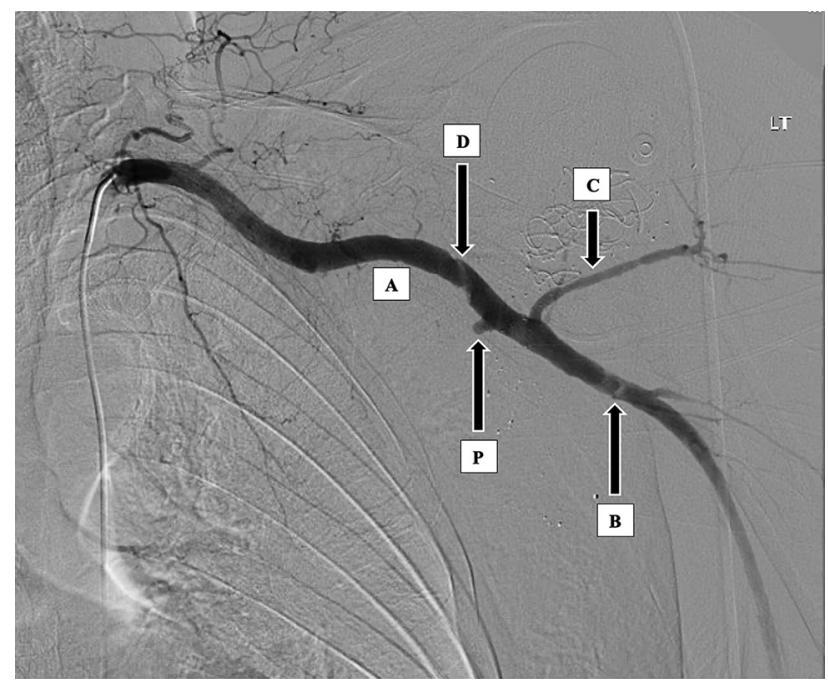

Figure 1 EVR of ASI due to a gunshot wound - before. An oblique lucency is visible through the mid axillary artery (A) at the lateral scapular margin, presumably representing a dissection flap (D). There is a $5 \mathrm{~mm}$ saccular outpouching $1 \mathrm{~cm}$ proximal to the circumflex humeral artery (C), likely a small axillary artery pseudoaneurysm (P). Curvilinear lucencies within the proximal left brachial artery (B) likely represent an intimal flap as well.

Figures 1 and 2 demonstrate an example of EVR used for emergent repair in the case of penetrating ASI with focal lesions. While EVR historically failed when the injured vessel was completely transected or had an associated hematoma, technical advances are making this increasingly surmountable [13]. Combined approaches that establish through-and-through access, such as antegrade femoral access with a retrograde brachial cutdown, can help overcome these aforementioned challenges.

\section{THE EVOLVING PARADIGM SHIFT IN THE MANAGEMENT OF ASI}

The management of arterial trauma, particularly of junctional arterial trauma such as ASI, is a rapidly evolving landscape. A retrospective review of 153 ASIs noted that from 2003 to 2013 rates of EVR increased from $5.3 \%$ to $22.2 \%$ despite the absolute numbers of ASIs per year remaining constant [1,4-5]. Meanwhile, the incidence of OSR decreased from $47 \%$ to $32 \%$ in a different study from 2002 to 2014 [3]. Danetz et al. also noted in a 2005 retrospective review of 46 ASIs that up to $50 \%$ of them could have been managed with EVR, but the actual proportion of EVR was much lower. $25 \%$ of those patients were hemodynamically unstable, which could explain that proportion receiving OSR. The indications for EVR are not uniform, and surgeon-preference/ ability is a major factor $[10,11]$. OSR is more likely to be selected by a surgeon who is not confident in EVR techniques or more comfortable with OSR. However, with the advent of ever-improving endovascular devices

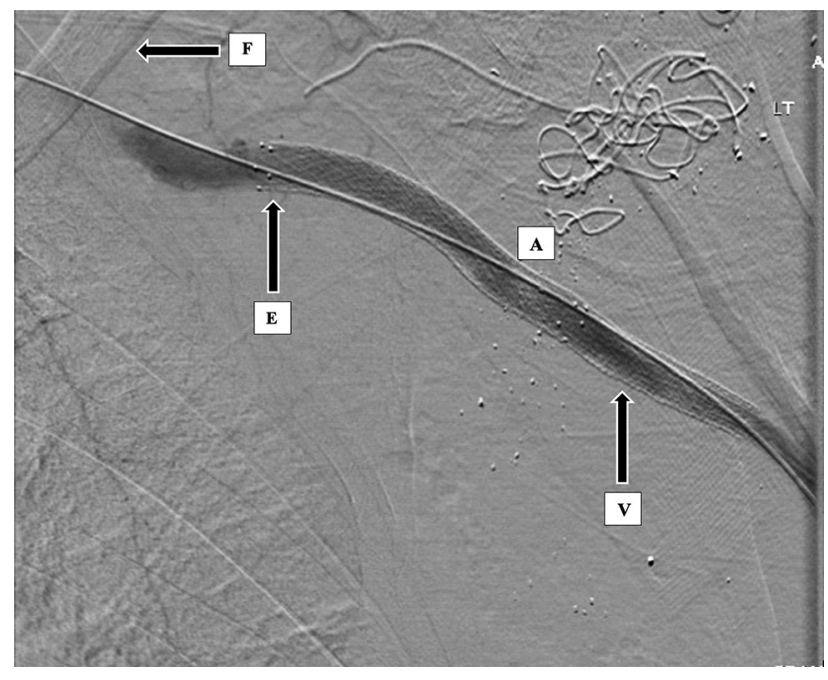

Figure 2 EVR of ASI due to a gunshot wound - after. The lateral margin of the first rib (F) is seen medially. Thereafter, the axillary artery (A) has two self-expanding Viabahn covered stents (V) placed within it, successfully covering the injured segments with no further arterial extravasation visible. An Epic self-expanding bare metal stent (E) has also been placed to exclude further medial extension of axillary artery injury.

and techniques, a paradigm shift towards EVR is gradually taking place.

\section{PATIENT SELECTION}

The selection of candidates for EVR instead of OSR has evolved in recent years. Anatomic considerations are first and foremost given the unique position and physical confinement of axillary and subclavian arteries. For injuries in an anatomically inaccessible segment of the vessel adjacent in the chest or posterior to the clavicle, EVR is often the best first-line option. When the injured segment is adjacent or posterior to the clavicle, OSR is particularly challenging due to the requirement for sternotomy or high anterolateral thoracotomy to obtain adequate exposure for proximal and distal control. However, EVR carries risks of stent fatigue and fracture given the stress of repetitive subclavian artery compression between the first rib and clavicle, and of the axillary artery at the junction of the thorax and upper arm [17,32].

When EVR is the chosen method of repair, fabric covered stents may be at risk of covering some branches of the subclavian artery causing ischemia. Despite this concern, collateral or first-order branch vessels have been safely sacrificed $[11,21]$ in prior reports, including the internal mammary artery (although this may be required in future coronary artery bypass grafting) and ostensibly non-dominant vertebral arteries. Whenever a vertebral artery ostium is at risk of being covered during EVR, the supra-aortic trunk must be carefully assessed for the presence of excellent cerebral primary collateral circulation. EVR may also be suboptimal for long segment 
Table 1 Comparative morbidity and relevant operative details for OSR versus EVR of ASI from select contemporary studies.

\begin{tabular}{lccc}
\hline & In OSR Cases & In EVR Cases & \\
\hline Morbidity & & & \\
$\quad$ Surgical site infection [3] & $7.1 \%$ & $4.2 \%$ & 0.03 \\
Pneumonia [3] & $8.3 \%$ & $5.1 \%$ & 0.03 \\
latrogenic brachial plexus injury [42] & $14.3 \%$ & $0 \%$ & $\mathrm{n} / \mathrm{a}$ \\
ICU admission [3] & $31 \%$ & $21 \%$ & 0.01 \\
Overall mortality [3] & $14.2 \%$ & $8.8 \%$ & 0.01 \\
Operative Details & & & $0.01[10]$ \\
Estimated blood loss, mean & $220 \mathrm{ml}$ ml & $0.03[15]$ \\
& $1225 \mathrm{ml}$ & $0.04[10]$ \\
Opperative time, mean & $193 \mathrm{~min}$ & $50 \mathrm{ml}$ & $132 \mathrm{~min}$ \\
& $230 \mathrm{~min}$ & $149 \mathrm{~min}$ & $0.03[15]$ \\
Length of stay, median [3] & 8 days & 4 days & 0.01 \\
\hline
\end{tabular}

injuries, as longer stent length may increase thrombotic risk and inadvertent coverage of small branches that could become collaterals later - this would essentially "burn bridges" and risk more severe ischemia from stent thrombosis than a failed surgical bypass [17].

\section{Hemodynamic Stability and Balloon Occlusion}

Patients that are hemodynamically unstable or receiving cardiopulmonary resuscitation are less likely to be deemed candidates for EVR [17]. They are also more likely to have multi-system injuries necessitating prompt open surgery rather than isolated endovascular intervention. Moreover, patients with large hematomas at risk of compressing the brachial plexus and leading to persistent neurologic deficits may also require OSR rather than EVR to evacuate it [13]. Whereas hemodynamic instability was previously cited as a contraindication to EVR, now, in the era of EVTM and hybrid ORs, it is an evolving indication [33-35]. With innovations such as resuscitative endovascular balloon occlusion of the aorta (REBOA) and proximal balloon occlusion of the subclavian artery, it is becoming more realistic to take unstable patients for hybrid or EVR [12,28] even with active extravasation and expanding hematoma [36]. External balloon catheter tamponade can also be helpful, for example, with a large (e.g. 20 cc) Foley balloon inserted through the bleeding injury tract and then inflated to the point of hemostasis. This measure for damage control is particularly invaluable for junctional injuries such as ASI [37] as it can transform an emergent situation into a controlled one. If able to cover the injury, a non-compliant angioplasty balloon could provide both proximal and distal control, thereby facilitating a hybrid solution. It warrants emphasis that endovascular techniques do not exclude OSR and can in fact be valuable adjuncts to it.

Despite the theoretical increase in bleeding proximal to the site of aortic occlusion, some authors support the use of EVR with REBOA [3,31,33,34], following basic resuscitation (blood product resuscitation, tube thoracostomy), and have published reports of positive outcomes thereafter [38]. Branco and DuBose reviewed 7 studies on REBOA and 10 on EVR of ASI, concluding that REBOA was a safe and effective alternative to open thoracotomy in critically ill trauma patients [31]. One head-to-head comparison from a large American trauma registry also posited there was no significant difference between REBOA and emergency thoracotomy [38]. If REBOA is utilized pre-procedure, further clinical deterioration can be rapidly temporized, and as this is now more commonplace in major trauma centers, it may contribute to the shift towards EVR of traumatic vascular injuries such as ASI as well. It has been postulated that the only absolute contraindication to EVR would be failure to cross the injured area with a wire [3], although it is also important to recognize that acute care providers who can use REBOA may not also be trained for more complex EVR of ASI.

\section{TECHNIQUES}

The techniques of EVR for ASI involve some consistent elements. It usually takes place in a hybrid operating room or a room with full endovascular suite capabilities. An anesthesiologist is present and general anesthesia is utilized. Antibiotic prophylaxis is administered, but most patients are not heparinized leading up to the procedure nor during it. Patients are prepped and draped for both endovascular and possible open repair.

Most cases start with percutaneous access via the common femoral artery, which can be ultrasound-guided if required. Percutaneous brachial access with a low profile 4-French introducer sheath is also an option, although it tends to be reserved as accessory access when dual access is required. If immediate proximal control of hemorrhage is required, selection of the injured vessel for the aortic arch is performed and balloon occlusion is 
performed. Commonly used balloons to do so include larger profile compliant aortic molding balloons, appropriate diameter semi-compliant or non-compliant angioplasty balloons, or lower profile REBOA specific occlusion devices.

A soft guidewire and diagnostic catheter is typically used to access the injured vessel and selective angiography is done to define the target lesion. This can also be used for device sizing (based on the normal parent vessel diameter) if not already planned using prior preoperative computed tomography angiography. Then, a long sheath (8-10 French) is typically inserted reaching just proximal to the site of injury if from the femoral access, and 4-5 French from the secondary (e.g. brachial access) if present. When the brachial artery is the primary approach for actual stent deployment, a brachial artery cutdown is often required to accommodate larger device and sheath sizes ( $>6$ French). Radial access is also becoming an option, with advances in lower profile endovascular systems. The retrograde transbrachial approach can be particularly helpful when a total occlusion exists or antegrade guidewire crossing of the injured segment is unsafe. Snaring of the wire to allow for through-and-through "bodyfloss" technique is often a helpful adjunct as well. Advancing the stent via brachial artery access alone if possible can potentially be safer, as it avoids the neurologic complications of negotiating aortic arch vessels, which can be anatomically variant or thrombus laden, with a large sheath and covered stent [13]. For the subclavian artery, the stent size is usually $6-8 \mathrm{~mm}$ (including oversizing the device by $10-20 \%$ or about $1 \mathrm{~mm}$ to ensure adequate seal) $\times 40 \mathrm{~mm}$ length [12,34]. Generally, either side of the stent-graft incorporates $1-2 \mathrm{~cm}$ of normal artery $[13,27,34]$. A completion angiogram is done, which confirms distal runoff through to the forearm.

If a hematoma continues to expand or hypotension persists after successful arterial stenting, concurrent venous injury (especially in the setting of penetrating trauma) is a crucial consideration. This can also be investigated either with a venogram or open surgical exploration.

Periprocedural complications to be aware of include local access site related events such as artery thrombosis, pseudoaneurysm or intimal flap development, nerve injury, compartment syndrome from hematoma (brachial access), phlebitis, and lymphangitis [31,39]. Arterial closure devices can be used if indicated. Remote complications such as embolic events, cerebral infarction, and one immediate peri-procedural mortality have also been reported [31,39].

\section{DEVICES}

The use of both balloon-expandable and self-expanding stents, generally oversized $10-15 \%$, covered with Dacron or PTFE have been used for ASI. Those that feature commonly in reported cases and series include the Viabahn (Gore and Associates, Flagstaff, AZ, USA), iCAST (Atrium Interventional, Hudson, New Hampshire,
USA), Covered Wall-Stent (Boston Scientific, Watertown, MA, USA), PTFE-covered Palmaz stent (Cordis Corporation, Miami Lakes, FL, USA), and Fluency stent (Bard, Murray Hill, NJ, USA). The choice of balloon-expandable versus self-expanding stent corresponds to the specific site of injury along the vessel. A key anatomic difference across the subclavian and axillary arteries is the extent of repetitive movements at the junction of the clavicle and first rib as well as the thorax and upper arm. This often precludes placement of a balloon-expanding stent at the thoracic outlet, distal subclavian or axillary arteries, as repeated movements increase their risk of stent fracture and thrombosis. For a proximal subclavian injury, on the other hand, balloon-expanding stents allow more deployment precision if landing close to other key vessels such as the vertebral artery ostium. Figure 2 illustrates an example of the use of both a Viabahn self-expanding covered stent and an Epic self-expanding bare metal stent. To date, no study has compared the patency of ASI stents by types or locations. Stent selection currently appears to be based on provider preference and appraisal of these key anatomic considerations.

Post-operative surveillance is generally accompanied by imaging of the entire axillosubclavian segment and its adjacent inflow and outflow arteries at regular intervals, initially at 1 month, 6 months, and then annually. This is typically accomplished by Duplex ultrasonography of the entire axillosubclavian segment. Patients receive varied durations of antiplatelet therapy thereafter, ranging from 1 month to lifelong [6,17,39]. Follow up is limited in most cases with a wide range, from none post-discharge to 6 years $[35,39]$.

\section{OUTCOMES}

Contemporary studies of the outcomes of OSR versus EVR of ASI suggest significantly lower in-hospital mortality in general. A head-to-head comparison across two high-volume American trauma centers of EVR and OSR that propensity-matched patients also showed that EVR reduced in-hospital mortality and rates of surgical site infections [12]. EVR causes less disruption of adjacent tissues, and reduces operative time and need for blood products $[15,40,41]$. Patients have lower rates of postoperative complications such as pneumonia, fewer ICU admissions, and shorter hospital length of stay, and overall EVR has been independently associated with improved survival [3]. Multiple series comparing the outcomes of EVR versus OSR for ASI have concluded that EVR involves reduced operative times and blood loss $[10,15]$ as well as lower rates of brachial plexus injury [42]. Table 1 summarizes these key results. One series of 27 ASIs demonstrated a $95 \%$ success rate with EVR, with the few failed attempts being due to stent deployment failure or inability to fully cover the lesion [10]. Therein, the 12 "endofeasible" lesions that successfully underwent EVR had similar 1-year arterial patency as OSR. 
An early retrospective review of 46 ASIs found only 21 that acutely received preoperative diagnostic arteriography, and among those only 3 had endovascular intervention. Two had EVR to treat injured first order subclavian artery branches, and one had an axillary artery transection for which proximal control was obtained with a 5 -mm balloon in the distal subclavian artery prior to OSR [11]. Of note, 6 patients with subclavian vascular injury underwent emergency department thoracotomy and none of them survived. A more recent retrospective review of EVR versus OSR outcomes for civilian arterial traumatic injuries from 2002 to 2014 showed the rate of EVR increasing from $3.1 \%$ to $8.9 \%$, which is a clear upward trajectory [3]. However, the same study still noted continued cautious selection because EVR patients were older, with higher systolic BP and lower heart rate on admission, and their time to repair was faster, all reflective of lower injury severity scores (ISS) [3]. Despite propensity score matching, confounders in patient selection of EVR over OSR inevitably make EVR outcomes appear more favorable as patients selected for EVR tend to have lower ISS and those who have OSR tend to start with more extensive traumatic injuries.

Primary patency of covered stents has been called the Achilles' heel of EVR [17], and the outcome data does remain limited to case reports and case series or retrospective reviews. These are rare injuries, and the population they occur in is especially challenging to follow up, leaving long-term outcomes after EVR a matter of ongoing concern. Primary patency of covered stents for ASI ranges from $69 \%$ to $88 \%[28,43]$. A large retrospective review on EVR of ASI found $84.4 \%$ stent patency for the duration of patient follow-up [39]. However, stent-related complications have been noted. In one retrospective study the rate was $2 / 15$ for a type $1 \mathrm{~A}$ endoleak and a stent thrombosis [12], which has also been reported in multiple large retrospective reviews [6,17]. Another small series of six ASIs resulted in 2/6 patients with persistent endoleaks requiring balloon angioplasty to improve stent apposition [34]. Although stent thrombosis is encountered at times, it does not always necessitate reintervention if asymptomatic [6]. Close follow-up, which may prevent this, has proven difficult to achieve in this patient population. Moreover, the burden of intensive long-term post-operative surveillance is not insignificant [32] and can be hard to maintain, particularly in younger patients. While it is too early for truly long-term outcomes of stent-grafting for ASI to be established, its utility even as a temporizing measure or bridge to later definitive repair $[6,17]$ is still important and may improve the outcomes of OSR done later in a non-emergent manner.

\section{CONCLUSION}

In patients with ASI, a junctional traumatic vascular injury in which OSR is highly morbid, EVR can be a safer alternative that achieves similar immediate results. With continually better tools and a paradigm shift towards more extensive endovascular training and hybrid operating suites, EVR is likely to grow with more well-trained practitioners comfortable with EVR of traumatic vascular injuries. As we enter an era of EVTM, vascular surgeons and interventional radiologists in many systems are increasingly likely to work collaboratively with trauma teams. The resulting paradigm shift towards EVR may notably improve morbidity and mortality for the young patients that suffer from these injuries even in extremis. Trauma registries may be an important alternative to randomized control trials for future research.

\section{Ethics Statement}

(1) All the authors mentioned in the manuscript have agreed to authorship, read and approved the manuscript, and given consent for submission and subsequent publication of the manuscript.

(2) The authors declare that they have read and abided by the JEVTM statement of ethical standards including rules of informed consent and ethical committee approval as stated in the article.

\section{Conflicts of Interest}

The authors declare that they have no conflicts of interest.

\section{Funding}

The authors received no financial support for the research, authorship, and/or publication of this article.

\section{Author Contributions}

All authors have made a substantial contribution to this article. They have all reviewed and approved the final submission draft.

\section{REFERENCES}

[1] Graham JM, Feliciano DV, Mattox KL, Beall Jr AC, DeBakey ME. Management of subclavian vascular injuries. J Trauma. 1980;20(7):537-44.

[2] McKinley AG, Carrim AA, Robbs JV. Management of proximal axillary and subclavian artery injuries. $\mathrm{Br} \mathrm{J}$ Surg. 2000;87(1):79-85.

[3] Romagnoli AN, Zeeshan M, Joseph B, Brenner ML. Utilization of endovascular and open surgical repair in the United States: a 10-year analysis of the National Trauma Databank (NTDB). Am J Surg. 2019;218(6):1128-33.

[4] Lin PH, Koffron AJ, Guske PJ, et al. Penetrating injuries of the subclavian artery. Am J Surg. 2003;185(6):580-4.

[5] Biagioni RB, Burihan MC, Nasser F, Biagioni LC, Ingrund JC. Endovascular treatment of penetrating arterial trauma with stent grafts. VASA Z Gefasskrankheiten. 2018;47(2):125-30. 
[6] Matsagkas M, Kouvelos G, Peroulis M, Xanthopoulos D, Bouris V, Arnaoutoglou E. Endovascular repair of blunt axillo-subclavian arterial injuries as the first line treatment. Injury. 2016;47(5):1051-6.

[7] Demetriades D, Chahwan S, Gomez H, et al. Penetrating injuries to the subclavian and axillary vessels. J Am Coll Surg. 1999;188(3):290-5.

[8] Hyre CE, Cikrit DF, Lalka SG, Sawchuk AP, Dalsing MC. Aggressive management of vascular injuries of the thoracic outlet. J Vasc Surg. 1998;27(5):880-4; discussion 884-5.

[9] du Toit D, Strauss D, Blaszczyk M, de Villiers R, Warren B. Endovascular treatment of penetrating thoracic outlet arterial injuries. Eur J Vasc Endovasc Surg. 2000;19(5): 489-95.

[10] Xenos ES, Freeman M, Stevens S, Cassada D, Pacanowski J, Goldman M. Covered stents for injuries of subclavian and axillary arteries. J Vasc Surg. 2003;38(3):451-4.

[11] Danetz JS, Cassano AD, Stoner MC, Ivatury RR, Levy MM. Feasibility of endovascular repair in penetrating axillosubclavian injuries: a retrospective review. J Vasc Surg. 2005;41(2):246-54.

[12] Branco BC, Boutrous ML, DuBose JJ, et al. Outcome comparison between open and endovascular management of axillosubclavian arterial injuries. J Vasc Surg. 2016;63(3):702-9.

[13] Shalhub S, Starnes BW, Tran NT. Endovascular treatment of axillosubclavian arterial transection in patients with blunt traumatic injury. J Vasc Surg. 2011;53(4): 1141-4.

[14] Starnes BW, Arthurs ZM. Endovascular management of vascular trauma. Perspect Vasc Surg Endovasc Ther. 2006;18(2):114-29.

[15] Shalhub S, Starnes BW, Hatsukami TS, Karmy-Jones R, Tran NT. Repair of blunt thoracic outlet arterial injuries: an evolution from open to endovascular approach. J Trauma. 2011 Nov;71(5):E114-121.

[16] DeBakey ME, Simeone FA. Battle injuries of the arteries in World War II: an analysis of 2,471 cases. Ann Surg. 1946;123(4):534.

[17] Chopra A, Modrall JG, Knowles M, Phelan HA, Valentine RJ, Chung J. Uncertain patency of covered stents placed for traumatic axillosubclavian artery injury. J Am Coll Surg. 2016;223(1):174-83.

[18] Demetriades D, Asensio JA. Subclavian and axillary vascular injuries. Surg Clin North Am. 2001;81(6):135773, xiii.

[19] Buscaglia LC, Walsh JC, Wilson JD, Matolo NM. Surgical management of subclavian artery injury. Am J Surg. 1987;154(1):88-92.

[20] Mousa A, Chong B, AbuRahma AF. Endovascular repair of subclavian/axillary artery injury with a covered stent. A case report and review of literature. Vascular. 2013; 21(6):400-4.

[21] Abouljoud MS, Obeid FN, Horst HM, Sorensen VJ, Fath JJ, Chung SK. Arterial injuries of the thoracic outlet: a ten-year experience. Am Surg. 1993;59(9):590-5.

[22] Kalakuntla V, Patel V, Tagoe A, Weaver W. Six-year experience with management of subclavian artery injuries. Am Surg. 2000;66(10):927-30; discussion 930-1.

[23] Dotter CT, Judkins MP. Transluminal treatment of arteriosclerotic obstruction. Circulation. 1964;30(5):654-70.
[24] Parodi JC, Palmaz JC, Barone HD. Transfemoral intraluminal graft implantation for abdominal aortic aneurysms. Ann Vasc Surg. 1991;5(6):491-9.

[25] Brandt MM, Kazanjian S, Wahl WL. The utility of endovascular stents in the treatment of blunt arterial injuries. J Trauma. 2001;51(5):901-5.

[26] Lyden SP, Srivastava SD, Waldman DL, Green RM. Common iliac artery dissection after blunt trauma: case report of endovascular repair and literature review. J Trauma. 2001;50(2):339-42.

[27] Kee-Sampson J, Eadie E, Matteo J, Shabandi N, Meyer T, Bashir S. Mind the gap: a novel endovascular technique to repair a transected vessel. Vascular. 2020;28(4): 436-40.

[28] Branco BC, DuBose JJ, Zhan LX, et al. Trends and outcomes of endovascular therapy in the management of civilian vascular injuries. J Vasc Surg. 2014;60(5):1297307.e1.

[29] Chang R, Fox EE, Greene TJ, et al. Multicenter retrospective study of noncompressible torso hemorrhage: anatomic locations of bleeding and comparison of endovascular versus open approach. J Trauma Acute Care Surg. 2017;83(1):11-8.

[30] Hörer T. Resuscitative endovascular balloon occlusion of the aorta (REBOA) and endovascular resuscitation and trauma management (EVTM): a paradigm shift regarding hemodynamic instability. Eur J Trauma Emerg Surg. 2018;44(4):487-9.

[31] Branco BC, DuBose JJ. Endovascular solutions for the management of penetrating trauma: an update on REBOA and axillo-subclavian injuries. Eur J Trauma Emerg Surg. 2016;42(6):687-94.

[32] Sinha S, Patterson BO, Ma J, et al. Systematic review and meta-analysis of open surgical and endovascular management of thoracic outlet vascular injuries. J Vasc Surg. 2013;57(2):547-567.e8.

[33] Martinez C, Maazoun Y, Durieux R, Defraigne J, Legrand V. Endovascular repair of the left subclavian artery after gunshot wound: an alternative to surgical treatment. Acta Cardiol. 2012;67(5):609-12.

[34] Cohen JE, Rajz G, Gomori JM, et al. Urgent endovascular stent-graft placement for traumatic penetrating subclavian artery injuries. J Neurol Sci. 2008;272(1):151-7.

[35] Altoijry A, Nouh T, Alburakan A, Ibrahim M, Altuwaijri TA. Endovascular repair of a penetrating axillary artery injury. Braz J Cardiovasc Surg. 2019;34(2):226-8.

[36] Elkbuli A, Shaikh S, McKenney M, Boneva D. Successful management with endovascular stent graft repair following gunshot wound to the subclavian artery: case report and literature review. Int J Surg Case Rep. 2019; 64:75-9.

[37] Roberts DJ, Bobrovitz N, Zygun DA, et al. Indications for use of thoracic, abdominal, pelvic, and vascular damage control interventions in trauma patients: a content analysis and expert appropriateness rating study. J Trauma Acute Care Surg. 2015;79(4):568-79.

[38] DuBose JJ, Scalea TM, Brenner M, et al. The AAST prospective Aortic Occlusion for Resuscitation in Trauma and Acute Care Surgery (AORTA) registry: data on contemporary utilization and outcomes of aortic occlusion and resuscitative balloon occlusion of the aorta (REBOA). J Trauma Acute Care Surg. 2016;81(3):409-19. 
[39] DuBose JJ, Rajani R, Gilani R, et al. Endovascular management of axillo-subclavian arterial injury: a review of published experience. Injury. 2012;43(11):1785-92.

[40] Schönholz CJ, Uflacker R, De Gregorio MA, Parodi JC. Stent-graft treatment of trauma to the supra-aortic arteries. A review. J Cardiovasc Surg (Torino). 2007;48(5):537-49.

[41] Saad NEA, Saad WEA, Davies MG, Waldman DL, Fultz PJ, Rubens DJ. Pseudoaneurysms and the role of minimally invasive techniques in their management. Radiographics. 2005;25 Suppl 1:S173-89.
[42] Carrick MM, Morrison CA, Pham HQ, et al. Modern management of traumatic subclavian artery injuries: a single institution's experience in the evolution of endovascular repair. Am J Surg. 2010;199(1):28-34.

[43] Villamaria CY, Eliason JL, Napolitano LM, Stansfield RB, Spencer JR, Rasmussen TE. Endovascular Skills for Trauma and Resuscitative Surgery (ESTARS) course: curriculum development, content validation, and program assessment. J Trauma Acute Care Surg. 2014;76(4): 929-35; discussion 935-6. 